Working Paper 13-26

Statistics and Econometrics Series 22

Julio 2013
Departamento de Estadística Universidad Carlos III de Madrid

Calle Madrid, 126 28903 Getafe (Spain)

Fax (34) 91 624-98-49

\title{
ALLOCATION POLICIES OF REDUNDANCIES IN TWO-PARALLEL-SERIES AND TWO-SERIES-PARALLEL SYSTEMS
}

\author{
Henry Laniado* and Rosa E. Lillo**
}

\begin{abstract}
In this paper comparisons of allocation policies of components in twoparallel-series systems with two types of components are provided with respect to both, the hazard rate and the reversed hazard rate orders. The main results indicate that the life of this kind of system is stochastically maximized by unbalancing as much as possible the two classes of components. We only assume that the two distributions implied in the model have proportional hazard rates. The same type of comparisons are also given for the dual model, the two-series-parallel systems but assuming that the distributions implied in the model have proportional reversed hazard rates, and therefore the final conclusion is the opposite; that is, the reliability of the system improves as the similarity between the two parallel subsystems increases.
\end{abstract}

Keywords: Parallel-series system, series-parallel system, redundancy, hazard rate order, reversed hazard rate order, proportional hazard rates.

* Department of Statistics, Universidad Carlos III de Madrid, 28911, Leganés, Madrid, Spain. e-mail: hlaniado@est-econ.uc3m.es (Henry Laniado).

** Department of Statistics, Universidad Carlos III de Madrid, 28903, Getafe, Madrid, Spain. e-mail: lillo@est-econ.uc3m.es (Rosa E. Lillo) 


\title{
Allocation policies of redundancies in two-parallel-series and two-series-parallel systems
}

\author{
Henry Laniado* and Rosa E. Lillo ${ }^{\dagger}$
}

July 25, 2013

\begin{abstract}
In this paper comparisons of allocation policies of components in two-parallel-series systems with two types of components are provided with respect to both, the hazard rate and the reversed hazard rate orders. The main results indicate that the life of this kind of system is stochastically maximized by unbalancing as much as possible the two classes of components. We only assume that the two distributions implied in the model have proportional hazard rates. The same type of comparisons are also given for the dual model, the two-series-parallel systems but assuming that the distributions implied in the model have proportional reversed hazard rates, and therefore the final conclusion is the opposite; that is, the reliability of the system improves as the similarity between the two parallel subsystems increases.
\end{abstract}

Key words Parallel-series system, series-parallel system, redundancy, hazard rate order, reversed hazard rate order, proportional hazard rates.

${ }^{*}$ H. Laniado , Departamento de Estadística, Universidad Carlos III de Madrid, C/de la universidad 3028911 Leganés, Madrid, Spain. (e-mail: henry.laniado@uc3m.es)

${ }^{\dagger}$ R.E. Lillo, Departamento de Estadística, Universidad Carlos III de Madrid, C/Madrid 126-128 28903 Getafe, Madrid, Spain. (e-mail: rosaelvira.lillo@uc3m.es) 


\section{Notation and Acronyms}

$a \wedge b$ minimum of $a$ and $b$

$a \vee b$ maximum of $a$ and $b$

$X_{i}, Y_{i}$ random variables of type $X$ and $Y$, respectively

$F, G$ distribution functions of $X$ and $Y$, respectively

$f, g$ density functions of $X$ and $Y$, respectively

$\bar{F}, \bar{G}$ survival functions of $X$ and $Y$, respectively

$r_{X}, r_{Y}$ hazard rate function of $X$ and $Y$, respectively

$\tilde{r}_{X}, \tilde{r}_{Y}$ reversed hazard rate function of $X$ and $Y$, respectively

$S_{k}$ random variable representing the lifetime of system

$F_{k}$ distribution function of $S_{k}$

$\bar{F}_{k}$ survival function of $S_{k}$

$f_{k}$ density function of $S_{k}$

$r_{k}$ hazard rate function of $S_{k}$

$\tilde{r}_{k}$ reversed hazard rate function of $S_{k}$

$\leq_{s t}$ usual stochastic order

$\leq_{h r}$ hazard rate order

$\leq_{r h r}$ reversed hazard rate order

$\leq_{l r}$ likelihood ratio order 


\section{Introduction}

This paper deals with optimal allocation of the components in a two-parallel-series system when two different types of components are avalaible. Figure 1 shows the system that we consider and that each parallel component is an $n$-series system. The reference ElNeweihi et al. [9] is a classic paper in this topic. We assume that in one system there are $n$ components whose lifetimes are i.i.d. random variables and the lifetimes of the $n$ components in the other system are i.i.d. random variables but with different distribution functions. It is easy to notice that the number of possible different allocations of the

$2 n$ components in the system is $\frac{n+1}{2}$ if $n$ is odd, or $\frac{n+2}{2}$ if $n$ is even. In this paper, we focus on allocation policies of the system components where the reliability of the system is compared in terms of the hazard rate and the reversed hazard rate order.

The first point to be addressed in this paper is comparable to an initial series system of $n$ components that is duplicated (after connecting it in parallel with another series system of $n$ components) in order to improve its reliability. In the literature, this option for improving the reliability of some system is called the redundancy at system level. The allocation of active redundancies is a well-known way to improve the reliability of a system. Redundancy can be made at system level (duplicate the system) or at component level (redundancies in parallel with each component).

Most studies on optimal allocation of active spares deal with performance comparisons at the component level or with performance comparisons between component level versus system level. The importance of the redundancy and allocation of spares in a coherent system has been initially studied in Boland et al. [3, 5]. The problem of how and where to allocate redundancies to the components has been extensively studied in the reliability literature. For example, Boland et al. [4] and Shaked and Shanthikumar [22] studied the allocation problem for series and parallel systems using the stochastic order as a criterion for comparison, whereas Singh and Misra [24] and Romera et al. [21] used the precedence order. Valdés and Zequeira [27] and more recently Li et al. [16] also provided optimal allocation but using the hazard rate order and the increasing concave order, respectively. For more general systems such as the $k$-out-of- $n$ systems, Mi [18] also investigated optimal allocation of redundancies.

On the other hand, there are many references studying which option is better: redundancy at system level or at component level. Barlow and Proschan ([2], page 24) stated a well known principle: redundancy at the component level is better than redundancy at system level if the lifetime components are independent random variables and considering the usual stochastic order as criterion for comparison. Boland and El Neweihi [6] obtained 
a similar result replacing the usual stochastic order by the hazard rate order and Singh and Singh [25] achieved the same conclusion for k-out-of-n systems with components and redundancies being i.i.d and considering the likelihood ratio order. Misra et al. [19] investigated if the redundancy at the component level is better than the redundancy at the system level in terms of the hazard rate order for coherent systems with nonmatching components and spares, and for coherent systems with matching components and spares, in terms of the likelihood ratio order. Recently, Brito et al. [7] compared allocation of redundancy at the component level versus system level in two-component series systems with nonmatching components and spares in terms of the likelihood ratio order. Some recent results on this topic are provided in Misra et al. [20], Valdés et al. [28], Li and Ding [15], Li et al. [16] and the references therein. However, there are situations in which it is only possible to improve the system reliability with redundancy at system level, that is, duplication of the system. This is the case of certain pipeline processor systems (see, for instance, Austin [1]) where spatial redundancy is provided by separate processors.

The novelty of the model that we introduce in this paper is the possibility that one can choose among $2 n$ components ( $n$ of each type) to configure the whole system. One practical situation that fits our model is, for instance, a factory with a production system in which the final product is obtained through a process of $n$ successive stages (or steps). Each stage depends on a machine but the full process is finished when a product is treated by the $n$ machines whose lifetimes are identically distributed random variables. Hence the machines form a series system. Suppose that, due to an increase in the demand, the factory decides to open another line of production and the decision maker needs to buy additional $n$ machines in order to develop the same task. The new $n$ machines can be different from the original ones (for example, they can be either newer or second-hand machines). Notice that the total production system stops when at least one machine of each line has failed. The manager has many possibilities to allocate the machines in both lines by combining new machines with old machines. A natural question is, what is the optimal configuration of machines in order to improve the reliability of the productivity time?. This paper will focus on the answer to this question.

There are only few results in the literature dealing with stochastic comparisons of alternative allocations in parallel-series systems. To our knowledge, the most relevant results on this topic may be found in Boland et al. [3], El-Neweihi et al. [9] and Shaked and Shanthikumar [22] where comparisons in the usual stochastic order are given. Hence, the main contribution of this paper is to provide optimal allocation policies in terms of the hazard rate order and of the reversed hazard rate order. The main results indicate that the life of this kind of system is stochastically maximized by unbalancing as much 
as possible the two classes of components. The constraint in the distributions is that they have proportional hazard rate functions. This conclusion is similar to that showed in Shaked and Shanthikumar [22] [Result 2.14(p)] but for a special parallel-series system, in terms of the usual stochastic order.

We have also investigated the dual problem; i.e, a two-series-parallel system where the two components of the series system are parallel systems of $n$ components (see Figure 3). We have also considered that there are two types of distribution functions implied in the system, each one associated to $n$ components. We provide stochastic comparisons among allocation policies in terms of the hazard rate order. The results are similar to those for the parallel-series system but the constraint in the distributions is that they have proportional reversed hazard rates, so the final conclusion is the opposite; that is, the reliability of the system improves as the similarity between the two parallel subsystem increases.

The paper is organized as follows. After introducing the preliminary definitions in Section III, we deal with the optimal allocation of components in the system considering the reversed hazard rate order in Section 4. Section 5 has the same structure as Section 4 but considering the hazard rate ordering. In Section 6, we briefly describe the direction of future research to prove the same results as in the two previous sections but with the likelihood ratio order. Indeed, we formulate a conjecture. Finally, in Section 7 we give a result for the dual case of the system considered.

\section{Preliminaries}

In this section, we include the mathematical formulation of the system displayed in Figure 1 and the definitions and tools that we need in future sections. Consider a series system with $n$ components performing a task, and denote by $X_{i}, i=1 \ldots, n$, the random variables representing the identically distributed lifetimes of such components with distribution function $F$. We assume that $n$ additional components are available to strengthen the lifetime of that series system, but at a system level where combination among initial components and additional components are allowed. Denote by $Y_{i}, i=1, \ldots, n$, their lifetimes whose common distribution function $G$ can differ from that of the $X_{i}$ 's. This description applies to the case in which the two types of components come from different production centers, or to the case in which one type is formed by new components and the other one is formed by used components. As usual, independence among the lifetimes is assumed. Note that different allocations are possible at system level by combining initial components with the new redundancies. Specifically, let $S_{k}$ denote the lifetime of the 
system when the up-series system has $k$ components of type $X$ and $n-k$ components of type $Y$. Figure 1 illustrates this situation.

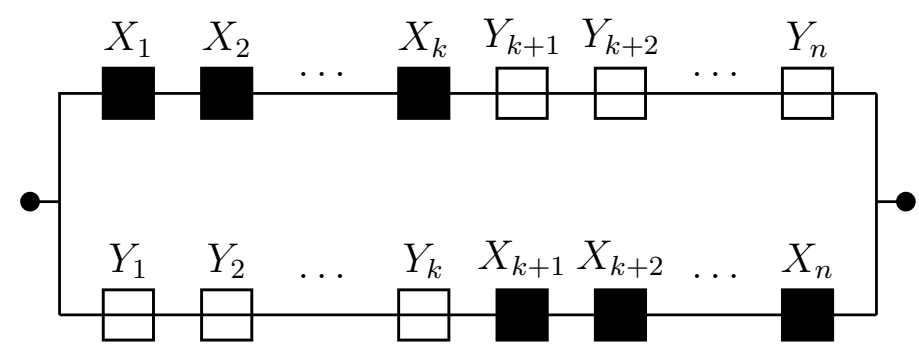

Figure 1: Two-parallel-series system with lifetime $\mathbf{S}_{\mathbf{k}}$

Then,

$$
S_{k}=\left(\bigwedge_{i=1}^{k} X_{i} \wedge \bigwedge_{i=k+1}^{n} Y_{i}\right) \vee\left(\bigwedge_{i=1}^{k} Y_{i} \wedge \bigwedge_{i=k+1}^{n} X_{i}\right)
$$

The objective of this paper is to find sufficient conditions to ensure stochastic comparisons between $S_{k_{1}}$ and $S_{k_{2}}$ for different values of $k_{1}, k_{2} \in\{0, \ldots, n\}$. In order to do that we rely on the following definitions of the stochastic orders considered in the paper.

Let $X$ and $Y$ be two absolutely continuous random variables that have common support $[0, \infty)$, distribution functions $F$ and $G$, and survival functions $\bar{F}=1-F$ and $\bar{G}=1-G$, respectively. Let $f$ and $g$ be their density functions, $r_{X}=f / \bar{F}$ and $r_{Y}=g / \bar{G}$ be their hazard rate functions and $\tilde{r}_{X}=f / F$ and $\tilde{r}_{Y}=g / G$ their reversed hazard rate functions, respectively.

We say that $X$ is smaller than $Y$

- in the usual stochastic order (denoted $X \leq_{s t} Y$ ) if, $\bar{F}(t) \leq \bar{G}(t)$ for all $t \in[0, \infty)$,

- in the hazard rate order (denoted $X \leq_{h r} Y$ ) if, $r_{X}(t) \geq r_{Y}(t)$ for all $t \in[0, \infty)$, or equivalently, if the ratio $\bar{F}(t) / \bar{G}(t)$ is non-increasing in $t \geq 0$, and

- in the reversed hazard rate order (denoted $X \leq_{r h r} Y$ ) if, $\tilde{r}_{X}(t) \leq \tilde{r}_{Y}(t)$ for all $t \in(0, \infty)$, or equivalently, if the ratio $F(t) / G(t)$ is non-increasing in $t>0$.

- in the likelihood ratio order (denoted $X \leq_{l r} Y$ ) if, the ratio $f(t) / g(t)$ is non-increasing in $t \geq 0$ as $X$ and $Y$ have the common support $[0, \infty)$.

For equivalent definitions and properties of the stochastic orders mentioned above, see Shaked and Shanthikumar [23]. 
The next notion will also be used in what follows. Given two random lifetimes, $X$ and $Y$, then they are said to follow the proportional hazard rate model (PHR) if $\bar{G}(t)=\bar{F}^{\alpha}(t)$ for all $t \geq 0$, and some $\alpha>0$. For examples and applications of these notions in system reliability see Valdés and Zequeira [26] and Kochar and Xu [[13],[14]], or in a different context, see for example, Finkelstein [10].

Given two vectors $\mathbf{a}=\left(a_{1}, a_{2}, \ldots, a_{n}\right)$ and $\mathbf{b}=\left(b_{1}, b_{2}, \ldots, b_{n}\right)$ and let $a_{[1]} \geq a_{[2]} \geq$ $\ldots \geq a_{[n]}$ and $b_{[1]} \geq b_{[2]} \geq \ldots \geq b_{[n]}$ the decreasing arrangements of the components of the two vectors, then the vector $\mathbf{a}$ is said to be majorized by the vector $\mathbf{b}$ in the Marshall and Olkin sense (denoted as $\mathbf{a} \prec \mathbf{b}$ ) if and only if, $\sum_{i=1}^{n} a_{[i]}=\sum_{i=1}^{n} b_{[i]}$ and $\sum_{i=1}^{k} a_{[i]} \leq \sum_{i=1}^{k} b_{[i]}$ for all $k=1,2 \ldots, n-1$. Besides, a function $\phi: I^{n} \subseteq \mathbb{R}^{n} \rightarrow \mathbb{R}$ is said to be a Schur-convex function if $\phi(\mathbf{a}) \leq \phi(\mathbf{b})$ whenever $\mathbf{a} \prec \mathbf{b}$. For extensive and comprehensive details on majorization order and Schur-convex functions, we refer the reader to the book of Marshall and Olkin [17].

\section{Comparison results in terms of the reversed hazard rate order}

If the usual stochastic order is considered and the random variables $X_{i}$ and $Y_{i}, i=1, \ldots, n$ are independent, then $S_{n-k_{1}} \geq_{s t} S_{n-k_{2}}$, for all $k_{1} \leq k_{2} \leq \frac{n}{2}$, regardless of the distributions $F$ and $G$. This result can be proved with a straightforward calculation of the survival functions of the two systems. A similar result has been formally derived in El-Neweihi et al. [9] for more general parallel-series systems. A similar conclusion was also reached by Shaked and Shanthikumar [22]. The most important conclusion is that the life of the parallel-series system is stochastically optimized when the heterogeneity between the series subsystems of the parallel system is maximized. If we consider the system of Figure 1 , this conclusion means that the system will have a higher reliability if the components and redundancies are as unbalanced as possible. We call this property as heterogeneity in the duplication of the system. Hence, the highest reliability will be obtained when each subsystem is composed by $n$ components of the same type, i.e., $k=0$.

In this paper, we prove that under the assumption of proportional hazard rate functions a stronger conclusion holds; i.e., the systems can be compared in terms of both, the hazard rate order and the reversed hazard rate order. In order to obtain the main result, we need two useful results. 
Lemma 4.1 The function $h(x)=\frac{x p^{x}}{1-p^{x}}$ is a convex function for $x>0$, and for all $p \in[0,1)$.

The second preliminary result is given in Marshall and Olkin [17], Proposition C.1., page 64.

Lemma 4.2 Let $c: I \subseteq \mathbb{R} \rightarrow \mathbb{R}$ be a convex function. Then the function

$$
\psi\left(x_{1}, \ldots, x_{n}\right)=\sum_{i=1}^{n} c\left(x_{i}\right)
$$

is Schur-convex on $I^{n}$.

The next statement shows conditions to ensure comparisons among the systems $S_{k}$ defined in (3.1) with respect to the reversed hazard rate order.

Theorem 4.3 Let $X$ and $Y$ have PHR, i.e., $\bar{G}(t)=\bar{F}^{\alpha}(t)$ for some positive $\alpha$, and for all $t \geq 0$. Then,

$$
S_{n-k_{1}} \geq_{r h r} S_{n-k_{2}}
$$

for every $k_{1} \leq k_{2} \leq \frac{n}{2}$

One conclusion here is that heterogeneity in the duplications improves the reversed hazard rate function of the system and as a consequence, the reliability of the system also improves. Hence, the optimal allocation will be obtained when both subsystems have the heterogeneity in the duplication of the system.

Note that the assumption of the previous theorem (PHR) is satisfied when $X$ and $Y$ are exponentially distributed with intensities $\lambda_{X}$ and $\lambda_{Y}$ and $\alpha=\frac{\lambda_{Y}}{\lambda_{X}}$. Indeed, this assumption is considered in many systems in Engineering and Medicine.

\section{Comparison results in terms of the hazard rate or- der}

The second main result describes conditions to obtain comparisons related to the random variables, $S_{k}, k=1, \ldots, n$, with respect to the hazard rate order. As in the previous Section, we need two auxiliary lemmas to prove the main result. 
Lemma 5.1 The function

$$
\gamma(x, p)=\frac{x p^{x-1}+(1-x) p^{-x}-1}{p^{x}+p^{1-x}-p},
$$

is a non-decreasing function in $x \in\left(0, \frac{1}{2}\right]$, for all $p \in(0,1]$.

Lemma 5.2 The function

$$
\varphi(p)=\frac{p^{\delta}+p^{1-\delta}-p}{p^{\beta}+p^{1-\beta}-p},
$$

is non-increasing in $p \in(0,1]$, for all $0<\delta \leq \beta \leq \frac{1}{2}$.

Proof. It is straightforward to see that $\varphi(p)$ is non-increasing in $p \in(0,1]$, for all $0<\delta \leq$ $\beta \leq \frac{1}{2}$, if

$$
\frac{\delta p^{\delta-1}+(1-\delta) p^{-\delta}-1}{p^{\delta}+p^{1-\delta}-p} \leq \frac{\beta p^{\beta-1}+(1-\beta) p^{-\beta}-1}{p^{\beta}+p^{1-\beta}-p},
$$

which holds from Lemma 5.1.

Now we can extend the statement of Theorem 4.3 but considering the hazard rate order.

Theorem 5.3 Let $X$ and $Y$ have PHR; i.e., $\bar{G}(t)=\bar{F}^{\alpha}(t)$ for some positive $\alpha$ and for all $t \geq 0$. Then

$$
S_{n-k_{1}} \geq_{h r} S_{n-k_{2}}
$$

for every $k_{1} \leq k_{2} \leq \frac{n}{2}$.

Proof. There is no loss of generality in assuming $\alpha \geq 1$. Then, we have that,

$$
\begin{aligned}
\bar{F}_{n-k}(t) & =1-\left[1-\bar{F}^{k}(t) \bar{G}^{n-k}(t)\right]\left[1-\bar{G}^{k}(t) \bar{F}^{n-k}(t)\right] \\
& =\bar{G}^{k}(t) \bar{F}^{n-k}(t)+\bar{G}^{n-k}(t) \bar{F}^{k}(t)-\bar{G}^{n}(t) \bar{F}^{n}(t) \\
& =\bar{F}^{n-k+\alpha k}(t)+\bar{F}^{k+\alpha(n-k)}(t)-\bar{F}^{(\alpha+1) n}(t) .
\end{aligned}
$$

Then, $S_{n-k_{1}} \geq_{h r} S_{n-k_{2}}$ if and only if the ratio

$$
\begin{aligned}
R(t) & =\frac{\bar{F}_{n-k_{1}}(t)}{\bar{F}_{n-k_{2}}(t)} \\
& =\frac{\bar{F}^{n-k_{1}+\alpha k_{1}}(t)+\bar{F}^{k_{1}+\alpha\left(n-k_{1}\right)}(t)-\bar{F}^{(\alpha+1) n}(t)}{\bar{F}^{n-k_{2}+\alpha k_{2}}(t)+\bar{F}^{k_{2}+\alpha\left(n-k_{2}\right)}(t)-\bar{F}^{(\alpha+1) n}(t)},
\end{aligned}
$$


is non-decreasing in $t \geq 0$. Denote $p(t)=\bar{F}^{(\alpha+1) n}(t)$. Obviously, $\frac{d p(t)}{d t} \leq 0$ and $0<p(t) \leq$ 1. Notice that (5.2) can be written as

$$
R_{p}(t)=\frac{p^{\delta}(t)+p^{1-\delta}(t)-p(t)}{p^{\beta}(t)+p^{1-\beta}(t)-p(t)}
$$

where

$$
\delta=\frac{\alpha k_{1}+n-k_{1}}{(\alpha+1) n} \quad \text { and } \quad \beta=\frac{\alpha k_{2}+n-k_{2}}{(\alpha+1) n} .
$$

Since $k_{1} \leq k_{2} \leq \frac{n}{2}$ implies that $\delta \leq \beta \leq \frac{1}{2}$, then $R_{p}$ decreases in $p$ by using Lemma 5.2. Since

$$
\frac{d}{d t} R(t)=\frac{d R_{p}(t)}{d p} \frac{d p(t)}{d t} \geq 0
$$

the proof is concluded.

As a consequence, heterogeneity in the duplications implies that the hazard rate function of the system decreases, and therefore the reliability of the system improves. Hence, as for the reversed hazard rate function, the optimal allocation will be also obtained when both subsystems are as different as possible.

\section{The likelihood ratio order}

Since the likelihood ratio order implies both the hazard rate order and the reversed hazard rate order (see Shaked and Shanthikumar [23]), one may wonder whether, under the same assumption of PHR imposed in Theorem 4.3 or Theorem 5.3, $S_{n-k_{1}}$ and $S_{n-k_{2}}$ are also ordered according to the likelihood ratio order. We are not able to answer this question analytically, but we outline a possible proof and we formulate a conjecture that if proven right, will conclude the proof.

Note that the density function of $S_{n-k}$ can be written as

$$
f_{n-k}(t)=\frac{f(t)}{\bar{F}(t)}\left[x_{1} \bar{F}^{x_{1}}(t)+x_{2} \bar{F}^{x_{2}}(t)-\left(x_{1}+x_{2}\right) \bar{F}^{x_{1}+x_{2}}(t)\right] \text {, }
$$

where

$$
x_{1}=k+\alpha(n-k) \quad \text { and } \quad x_{2}=\alpha k+n-k .
$$

Thus, considering $k_{1} \leq k_{2} \leq \frac{n}{2}$,

$$
S_{n-k_{1}} \geq_{l r} S_{n-k_{2}},
$$


if only if,

$$
\begin{aligned}
& \frac{f_{n-k_{1}}(t)}{f_{n-k_{2}}(t)} \\
& =\frac{x_{1}^{(1)} \bar{F}^{x_{1}^{(1)}}(t)+x_{2}^{(1)} \bar{F}^{x_{2}^{(1)}}(t)-\left(x_{1}^{(1)}+x_{2}^{(1)}\right) \bar{F}^{x_{1}^{(1)}+x_{2}^{(1)}}(t)}{x_{1}^{(2)} \bar{F}^{x_{1}^{(2)}}(t)+x_{2}^{(2)} \bar{F}^{x_{2}^{(2)}}(t)-\left(x_{1}^{(2)}+x_{2}^{(2)}\right) \bar{F}^{x_{1}^{(2)}+x_{2}^{(2)}}(t)}
\end{aligned}
$$

is non-decreasing in $t$, where

$$
x_{1}^{(i)}=k_{i}+\alpha\left(n-k_{i}\right) \text { and } x_{2}^{(i)}=\alpha k_{i}+n-k_{i}, \quad i=1,2 .
$$

By setting $u=\bar{F}(t),(6.2)$ is equivalent to requiring that the ratio

$$
\frac{x_{1}^{(1)} u^{x_{1}^{(1)}}+x_{2}^{(1)} u^{x_{2}^{(1)}}-\left(x_{1}^{(1)}+x_{2}^{(1)}\right) u^{x_{1}^{(1)}+x_{2}^{(1)}}}{x_{1}^{(2)} u^{x_{1}^{(2)}}+x_{2}^{(2)} u^{x_{2}^{(2)}}-\left(x_{1}^{(2)}+x_{2}^{(2)}\right) u^{x_{1}^{(2)}+x_{2}^{(2)}}}
$$

be non-increasing in $u \in(0,1]$. Let now denote

$$
\delta=\frac{x_{2}^{(1)}}{x_{1}^{(1)}+x_{2}^{(1)}} \quad \text { and } \beta=\frac{x_{2}^{(2)}}{x_{1}^{(2)}+x_{2}^{(2)}} .
$$

Under the assumption of $k_{1} \leq k_{2} \leq \frac{1}{2}$ and $\alpha \geq 1$ it holds $0 \leq \delta \leq \beta \leq \frac{1}{2}$. Now, if we let

$$
y=u^{x_{1}^{(1)}+x_{2}^{(1)}}=u^{x_{1}^{(2)}+x_{2}^{(2)}}=u^{(\alpha+1) n}
$$

then (6.3) is non-increasing in $u$ if and only if

$$
\frac{\delta y^{\delta}+(1-\delta) y^{1-\delta}-y}{\beta y^{\beta}+(1-\beta) y^{1-\beta}-y}
$$

is non-increasing in $y \in(0,1]$, for $0 \leq \delta \leq \beta \leq \frac{1}{2}$. Even though we have empirical evidence of this fact as shown in Figure 2 for some values of $\delta$ and for $\beta=0.5$, so far we have not been able to obtain a mathematical proof of this result. Thus the likelihood ratio comparison $S_{n-k_{1}} \geq_{l r} S_{n-k_{2}}$ remains as a conjecture in the paper. 


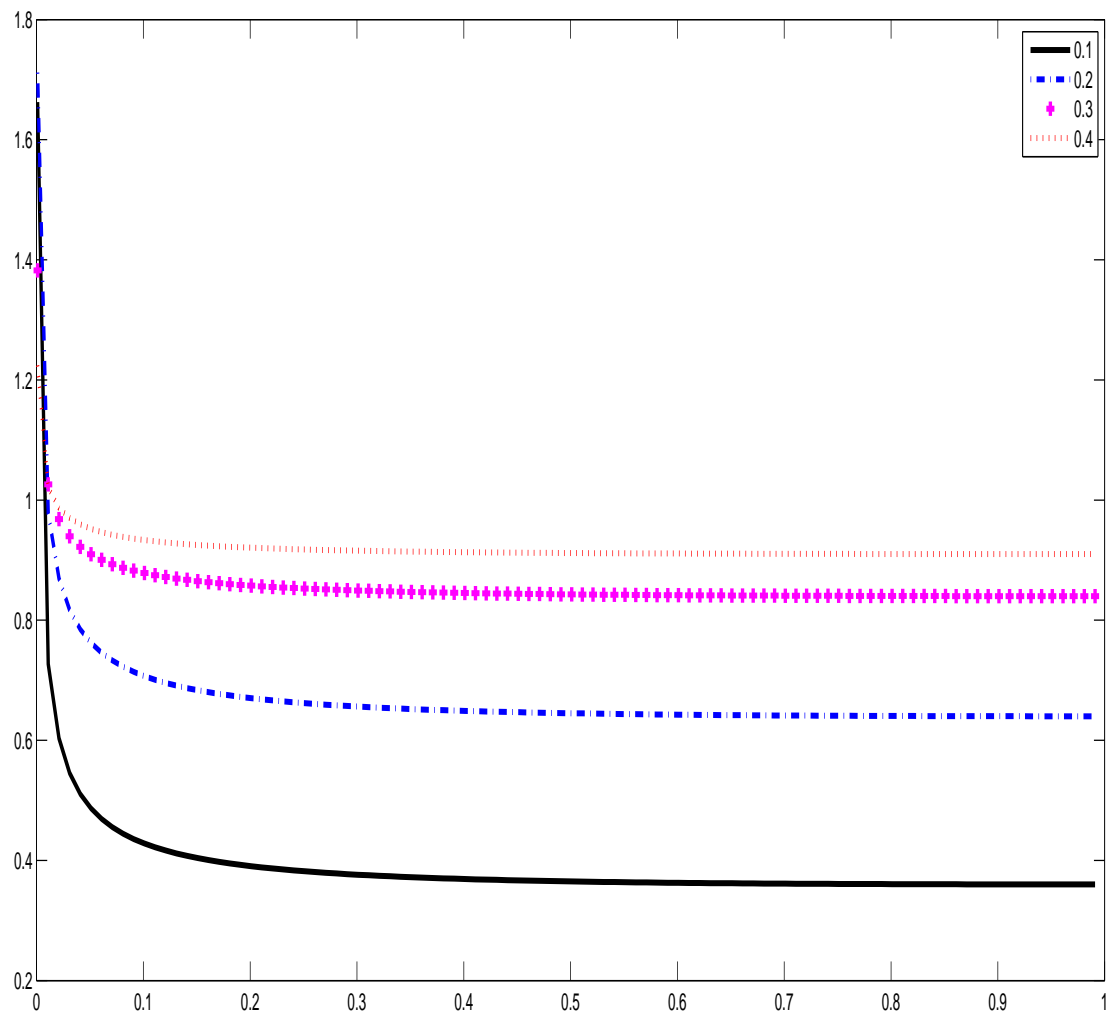

Figure 2: Graphic of (6.4) for different $\delta$-values and for $\beta=0.5$

\section{A result for the dual case}

Now consider the dual version of the system displayed in Figure 1; that is, a series-parallel (see El-Neweihi et al. [9]). We assume a two-series-parallel system which is formed by two subsystems connected in series, where each subsystem is formed by $n$ components in parallel. The new situation is represented in Figure 3. 


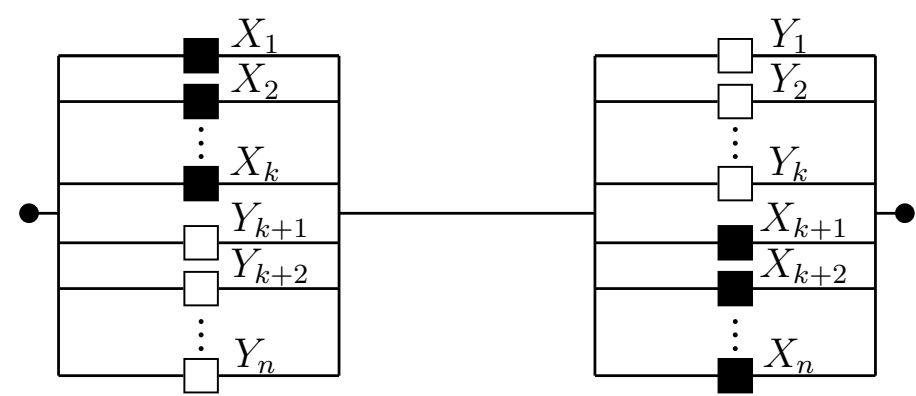

Figure 3: Two-series-parallel system with lifetime $\mathbf{S}_{\mathbf{k}}^{*}$

As mentioned in the Introduction, we have again $n$ components of kind $X$, and $n$ components of kind $Y$, with different distribution functions, $F$ and $G$, respectively. Let $S_{k}^{*}$ denote the lifetime of the system when the components are allocated in such a way that one of the parallel subsystems has $k$ components of kind $X$ and $n-k$ of kind $Y$. Then,

$$
S_{k}^{*}=\left(\bigvee_{i=1}^{k} X_{i} \vee \bigvee_{i=k+1}^{n} Y_{i}\right) \wedge\left(\bigvee_{i=1}^{k} Y_{i} \vee \bigvee_{i=k+1}^{n} X_{i}\right)
$$

We now assume that $X$ and $Y$ satisfy the proportional reversed hazard rate model (PRHR) which implies that $G(t)=F^{\alpha}(t)$ for all $t>0$ and some $\alpha>0$. For examples and applications of the reversed proportional hazard rate function notions in reliability of systems see Gupta et al. [12], Di Crescenzo [8], Gupta and Gupta [11], and the references therein. Again, recall that it is possible to combine components of each type in both subsystems. The following result states stochastic comparisons among $S_{k}^{*}, k=1, \ldots, n$ in terms of the hazard rate order.

Theorem 7.1 Let $X$ and $Y$ have PRHR; i.e., $G(t)=F^{\alpha}(t)$, for some positive $\alpha$ and for all $t>0$. Then,

$$
S_{n-k_{1}} \leq_{h r} S_{n-k_{2}}
$$

for every $k_{1} \leq k_{2} \leq \frac{n}{2}$

Proof. Without loss of generality we can assume $\alpha \geq 1$. For $k \leq \frac{n}{2}$ let us denote with $\bar{F}_{n-k}^{*}$ the survival function of $S_{n-k}^{*}$, i.e.,

$$
\begin{aligned}
\bar{F}_{n-k}^{*}(t) & =\left[1-F^{k}(t) G^{n-k}(t)\right]\left[1-G^{k}(t) F^{n-k}(t)\right] \\
& =\left[1-F^{k+\alpha(n-k)}(t)\right]\left[1-F^{\alpha k+n-k}(t)\right] .
\end{aligned}
$$


Then, the corresponding hazard rate function is

$$
\begin{aligned}
r_{n-k}^{*}(t) & =-\frac{d}{d t}\left[\ln \bar{F}_{n-k}^{*}(t)\right] \\
& =-\frac{d}{d t}\left[\ln \left[1-F^{k+\alpha(n-k)}(t)\right]+\ln \left[1-F^{\alpha k+n-k}(t)\right]\right] \\
& =\frac{f(t)}{F(t)}\left[\frac{(k+\alpha(n-k)) F^{k+\alpha(n-k)}(t)}{1-F^{k+\alpha(n-k)}(t)}\right] \\
& +\frac{f(t)}{F(t)}\left[\frac{(\alpha k+n-k) F^{\alpha k+n-k}(t)}{1-F^{\alpha k+n-k}(t)}\right] \\
& =\frac{f(t)}{F(t)} \sum_{i=1}^{2} \frac{x_{i} F^{x_{i}}(t)}{1-F^{x_{i}}(t)}
\end{aligned}
$$

where $x_{1}$ and $x_{2}$ are given in (6.1). Observe that both $x_{1}$ and $x_{2}$ are non-negative for all $\alpha \geq 1$. Let us denote

$$
x_{1}^{(i)}=k_{i}+\alpha\left(n-k_{i}\right) \text { and } x_{2}^{(i)}=\alpha k_{i}+n-k_{i}, \quad i=1,2 .
$$

Note that, for all $i=1,2$

$$
x_{1}^{(i)} \geq x_{2}^{(i)} ; \quad x_{1}^{(1)}+x_{2}^{(1)}=x_{1}^{(2)}+x_{2}^{(2)}=(\alpha+1) n
$$

and that $x_{1}^{(1)} \geq x_{1}^{(2)}$ whenever $k_{1} \leq k_{2} \leq \frac{n}{2}$, and $\alpha \geq 1$. These considerations imply that,

$$
\left(x_{1}^{(1)}, x_{2}^{(1)}\right) \succ\left(x_{1}^{(2)}, x_{2}^{(2)}\right) \text {, for all } k_{1} \leq k_{2} \leq \frac{n}{2} .
$$

Using Lemma 4.1 and Lemma 4.2, we have that the function

$$
\psi_{t}\left(x_{1}, x_{2}\right)=\sum_{i=1}^{2} \frac{x_{i} F^{x_{i}}(t)}{1-F^{x_{i}}(t)}
$$

is a Schur-convex function. Then, it follows from (7.4) that

$$
\begin{aligned}
r_{n-k_{1}}^{*}(t) & =\frac{f(t)}{F(t)} \psi_{t}\left(x_{1}^{(1)}, x_{2}^{(1)}\right) \\
& \geq \frac{f(t)}{F(t)} \psi_{t}\left(x_{1}^{(2)}, x_{2}^{(2)}\right) \\
& =r_{n-k_{2}}^{*}(t),
\end{aligned}
$$

for all $t \geq 0$ and then, the Theorem is proved.

An important conclusion is that the hazard rate function of the two-series-parallel system decreases as a parallel subsystem resembles another parallel subsystem, and as a consequence the reliability improves. In other words, the optimal allocation will be when both subsystems are as similar as possible. Notice that as we can see, this conclusion is the opposite to that obtained for the two-parallel-series system. 


\section{Appendix}

\section{A. Proof of Lemma 4.1}

The lemma trivially holds true for $p=0$. Now consider that $0<p<1$ and observe that

$$
\begin{aligned}
\frac{d^{2} h(x)}{d x^{2}} & =\ln p \frac{p^{x}}{\left(1-p^{x}\right)^{3}}\left[p^{x}(x \ln p-2)+x \ln p+2\right] \\
& =\ln p \frac{p^{x}}{\left(1-p^{x}\right)^{3}} l_{1}(x, p)
\end{aligned}
$$

where

$$
l_{1}(x, p)=p^{x}(x \ln p-2)+x \ln p+2 .
$$

To prove the convexity of $h(x)$, we need to show that $l_{1}(x, p) \leq 0$ for all $x>0$. Therefore, note that

$$
\begin{aligned}
\frac{\partial l_{1}(x, p)}{\partial x} & =p^{x}\left(x \ln ^{2} p-\ln p\right)+\ln p \\
& =\ln p\left[p^{x} x \ln p-p^{x}+1\right]=\ln p \tilde{l}(x, p),
\end{aligned}
$$

where

$$
\tilde{l}(x, p)=p^{x} x \ln p-p^{x}+1 .
$$

Given $x>0, \tilde{l}(x, p)$ is non-increasing in $p \in(0,1)$ since

$$
\frac{\partial \tilde{l}(x, p)}{\partial p}=x^{2} p^{x-1} \ln p \leq 0
$$

Thus,

$$
\tilde{l}(x, p) \geq \lim _{p \rightarrow 1^{-}} \tilde{l}(x, p)=0
$$

for all $0<p<1$.

It follows that

$$
\frac{\partial l_{1}(x, p)}{\partial x}=\ln p \tilde{l}(x, p) \leq 0,
$$

i.e., $l_{1}(x, p)$ is non-increasing in $x>0$, which implies that,

$$
l_{1}(x, p) \leq \lim _{x \rightarrow 0^{+}} l_{1}(x, p)=0, \quad \text { for all } x>0 .
$$

Hence, the convexity of $h$ follows (see 7.5). 


\section{B. Proof of Theorem 4.3}

Without loss of generality we can assume $\alpha \geq 1$. For $k \leq \frac{n}{2}$, let $F_{n-k}$ denote the distribution function of $S_{n-k}$, which can be written as,

$$
\begin{aligned}
F_{n-k}(t) & =\left[1-\bar{F}^{k}(t) \bar{G}^{n-k}(t)\right]\left[1-\bar{G}^{k}(t) \bar{F}^{n-k}(t)\right] \\
& =\left[1-\bar{F}^{k+\alpha(n-k)}(t)\right]\left[1-\bar{F}^{\alpha k+n-k}(t)\right] .
\end{aligned}
$$

Then, the corresponding reversed hazard rate function is given as

$$
\begin{aligned}
\tilde{r}_{n-k}(t)= & \frac{d}{d t}\left[\ln F_{n-k}(t)\right] \\
= & \frac{d}{d t}\left[\ln \left[1-\bar{F}^{k+\alpha(n-k)}(t)\right]+\ln \left[1-\bar{F}^{\alpha k+n-k}(t)\right]\right] \\
= & \frac{f(t)}{\bar{F}(t)}\left[\frac{(k+\alpha(n-k)) \bar{F}^{k+\alpha(n-k)}(t)}{1-\bar{F}^{k+\alpha(n-k)}(t)}\right] \\
& +\frac{f(t)}{\bar{F}(t)}\left[\frac{(\alpha k+n-k) \bar{F}^{\alpha+n-k}(t)}{1-\bar{F}^{\alpha k+n-k}(t)}\right] \\
= & \frac{f(t)}{\bar{F}(t)} \sum_{i=1}^{2} \frac{x_{i} \bar{F}^{x_{i}}(t)}{1-\bar{F}^{x_{i}}(t)},
\end{aligned}
$$

where $x_{1}, x_{2}$ are as in (6.1).

Observe that both $x_{1}$ and $x_{2}$ are non-negative for all $\alpha \geq 1$. Denote

$$
x_{1}^{(i)} \text { and } x_{2}^{(i)} \quad i=1,2, \text { as in (7.3). }
$$

Recall, from (7.4), that

$$
\left(x_{1}^{(1)}, x_{2}^{(1)}\right) \succ\left(x_{1}^{(2)}, x_{2}^{(2)}\right) \text {, for all } k_{1} \leq k_{2} \leq \frac{n}{2} .
$$

Using Lemma 4.1 and Lemma 4.2, we have that the function

$$
\bar{\psi}_{t}\left(x_{1}, x_{2}\right)=\sum_{i=1}^{2} \frac{x_{i} \bar{F}^{x_{i}}(t)}{1-\bar{F}^{x_{i}}(t)}
$$


is a Schur-convex function. Then, it follows from (7.8) that

$$
\begin{aligned}
\tilde{r}_{n-k_{1}}(t) & =\frac{f(t)}{\bar{F}(t)} \bar{\psi}_{t}\left(x_{1}^{(1)}, x_{2}^{(1)}\right) \\
& \geq \frac{f(t)}{\bar{F}(t)} \bar{\psi}_{t}\left(x_{1}^{(2)}, x_{2}^{(2)}\right) \\
& =\tilde{r}_{n-k_{2}}(t),
\end{aligned}
$$

for all $t \geq 0$ and then, the Theorem is proved.

C. Proof of Lemma 5.1

Consider

$$
\phi(x, p)=\ln \left(p^{x}+p^{1-x}-p\right) .
$$

It is easy to see that $\gamma(x, p)$ is non-decreasing in $x \in\left(0, \frac{1}{2}\right]$, for all $0<p \leq 1$, if

$$
\frac{\partial}{\partial x}\left[\frac{\partial \phi(x, p)}{\partial p}\right] \geq 0
$$

By symmetry of the second derivatives, we have that

$$
\begin{aligned}
\frac{\partial}{\partial x}\left[\frac{\partial \phi(x, p)}{\partial p}\right] & =\frac{\partial}{\partial p}\left[\frac{\partial \phi(x, p)}{\partial x}\right] \\
= & \frac{\partial}{\partial p}\left[\frac{p^{x}-p^{1-x}}{p^{x}+p^{1-x}-p} \ln p\right] \\
& =\frac{l_{2}(x, p)}{p}+\frac{\partial l_{2}(x, p)}{\partial p} \ln p
\end{aligned}
$$

where

$$
l_{2}(x, p)=\frac{p^{x}-p^{1-x}}{p^{x}+p^{1-x}-p} .
$$

Clearly,

$$
\frac{\partial l_{2}(x, p)}{\partial p}=\frac{-p^{x}}{\left(p^{2 x}-p^{x+1}+p\right)^{2}} Q(x, p),
$$

where

$$
Q(x, p)=(x-1) p^{2 x}+2(1-2 x) p^{x}+x p .
$$

For any $x \in\left(0, \frac{1}{2}\right]$, notice that

$$
\frac{\partial^{2} Q(x, p)}{\partial p^{2}}=2 x p^{x-2}(x-1)(2 x-1)\left(p^{x}-1\right)
$$


is non-positive in $0<p \leq 1$. Therefore,

$$
\frac{\partial Q(x, p)}{\partial p}=2 x(x-1) p^{2 x-1}+2 x(1-2 x) p^{x-1}+x
$$

is non-increasing in $p \in(0,1]$, which implies,

$$
\frac{\partial Q(x, p)}{\partial p} \geq\left[\frac{\partial Q(x, p)}{\partial p}\right]_{p=1}=x-2 x^{2} \geq 0,
$$

since $x \in\left(0, \frac{1}{2}\right]$. Hence, $Q(x, p)$ is non-decreasing in $p \in(0,1]$. Therefore,

$$
Q(x, p) \geq \lim _{p \rightarrow 0^{+}} Q(x, p)=0
$$

and as consequence, we have that $\frac{\partial l_{2}(x, p)}{\partial p} \leq 0$, for all $0<p \leq 1$. Since $l_{2}(x, p) \geq 0$ in $x \in\left(0, \frac{1}{2}\right]$, for all $0<p \leq 1$, the assertion follows from (7.9).

\section{Acknowledgment}

We want to thank to Franco Pellerey and Antonio Di Crescenzo for suggesting the main result of the paper. The authors of the paper would like to acknowledge the financial support by MEC project ECO2012-38442 and the project 2009/00035/001. 


\section{References}

[1] T.M. Austin, "DIVA: a reliable substrate for deep submicron microarchitecture design," In: Proceedings of the 32nd annual ACM/IEEE International Symposium on Microarchitecture, 196-207, IEEE Computer Society, Washington, DC, USA, 1999.

[2] R. E. Barlow and F. Proschan, Statistical Theory of Reliability and Life Testing, To Begin With, Silver Spring, 1981.

[3] P.J. Boland, E. El-Neweihi and F. Proschan, "Active redundancy allocation in coherent systems," Probability in the Engineering and Informational Sciences, 2, pp. 343-353, 1988.

[4] P.J. Boland, E. El-Neweihi and F. Proschan, "Stochastic order for redundancy allocations in series and parallel systems," Advances in Applied Probability, 24, pp. 161-171, 1992.

[5] P.J. Boland, E. El-Neweihi and F. Proschan, "Redundancy importance and allocation of spares in coherent systems," Journal of Statistical Planning and Inference, 29, pp. 55-65, 1991.

[6] P.J. Boland and E. El-Neweihi, "Component redundancy vs system redundancy in the hazard rate ordering," IEEE Transactions on Reliability, 44, pp. 614-619, 1995.

[7] G. Brito, R.I. Zequeira, and J.E. Valdés, "On the hazard rate and reversed hazard rate orderings in two-component series systems with active redundancies," Statistics E Probability Letters, 81, pp. 201-206, 2011.

[8] A. Di Crescenzo, "Some results on the proportional reversed hazards model," Statistics \& Probability Letters, 50, pp. 313-321, 2000.

[9] E. El-Neweihi, F. Proschan and J. Sethuraman, "Optimal allocation of components in parallel-series and series-parallel systems," Journal of Applied Probability, 23, pp. 770-777, 1986.

[10] M. Finkelstein, Failure Rate Modelling for Reliability and Risk,. Springer, London, 2008.

[11] R.C. Gupta and R.D. Gupta, "Proportional reversed hazard rate model and its applications," Journal of Statistical Planning E Inference, 137, pp. 3525-3536, 2007. 
[12] R.C. Gupta, R.D. Gupta and P.L. Gupta, "Modeling failure time data by Lehman alternatives," Communications in Statistics - Theory and Methods, 27, pp. 887-904, 1998.

[13] S.C. Kochar and M. Xu, "Some recent results on stochastic comparisons and dependence among order statistics in the case of PHR model," Journal of Iranian Statistical Society, 6, pp. 125-140, 2007.

[14] S.C. Kochar and M. Xu, "Stochastic comparisons of parallel systems when components have proportional hazard rates," Probability in the Engineering and Informational Sciences, 21, pp. 597-609, 2007.

[15] X. Li and W. Ding, "Optimal allocation of active redundancies to $k$-out-of- $n$ systems with heterogeneous components," Journal of Applied Probability, 47, pp. 254-263, 2010 .

[16] X. Li, R. Yan and X. Hu, "On the allocation of redundancies in series and parallel systems," Communications in Statistics - Theory and Methods, 40, pp. 959-968, 2011.

[17] A.W. Marshall and W. Olkin, Inequalities: Theory of Majorization and its Applications, Academic Press, New York, 1979.

[18] J. Mi, "Optimal active redundancy allocation in $k$-out-of- $n$ system," Journal of Applied Probability, 36, pp. 927-933, 1999.

[19] N. Misra, I.D. Dhariyal and N. Gupta, "Optimal allocation of active spares in series systems and comparison of component and system redundancies," Journal of Applied Probability 46, 19-34, pp. 2009.

[20] N. Misra, A.K. Misra and I.D. Dhariyal, "Active redundncy allocations in series systems," Probability in the Engineering and Informational Sciences, 25, pp. 219-235, 2011.

[21] R. Romera, J.E. Valdés and R.I. Zequeira, " Active-Redundancy allocation in systems," IEEE Transactions on Reliability, 53, pp. 313-318, 2004.

[22] M. Shaked and J.G. Shanthikumar, "Optimal allocation of resources to nodes of parallel and series systems," Advances in Applied Probability, 24, pp. 894-914, 1992.

[23] M. Shaked and J.G. Shanthikumar, 2007. Stochastic Orders, Springer: New York, 2007. 
[24] H. Singh and N. Misra, "On redundancy allocations in systems," Journal of Applied Probability, 31, pp. 1004-1014, 1994.

[25] H. Singh and R.S. Singh, "On allocation of spares at component level vs system level," Journal of Applied Probability, 34, pp. 283-287, 1997.

[26] J.E. Valdés and R.I. Zequeira, "On the optimal allocation of an active redundanciy in a two component series system," Statistics \& Probability Letters, 63, pp. 325-332, 2003.

[27] J.E. Valdés and R.I. Zequeira, "On the optimal allocation of two active redundancies in a two component series system," Operations Research Letters, 34, pp. 49-52, 2006.

[28] J.E. Valdés, G. Arango, R.I. Zequeira, and G. Brito, "Some stochastic comparisons in series systems with active redundancy," Statistics $\&$ Probability Letters, 80, pp. 945-949, 2010. 